\title{
Nucleon structure studies with the PANDA experiment at FAIR
}

\author{
Alaa Dbeyssi ${ }^{1, *}$ (on behalf of PANDA collaboration) \\ ${ }^{1}$ Helmholtz-Institut Mainz, Germany
}

\begin{abstract}
The PANDA experiment is one of the major projects in preparation at the upcoming FAIR facility in Darmstadt, Germany. A multipurpose high energy physics detector is currently under construction and will be operated at the High Energy Storage Ring of FAIR. High intensity antiproton beams will be available in the momentum range between $1.5 \mathrm{GeV} / c$ and $15 \mathrm{GeV} / c$ and will allow to address a broad physics program including hadron spectroscopy, study of charm and strangeness in nuclei, hypernuclear physics and other QCD topics. In addition, the PANDA experiment will offer unique possibilities to investigate the structure of the proton using different electromagnetic processes. In this contribution, the PANDA physics program related to the nucleon structure aspects is discussed. Feasibility studies of electromagnetic processes for the measurements of proton electromagnetic form factors in the time-like region and the nucleon-to-meson transition distribution amplitudes at PANDA, are reported.
\end{abstract}

\section{Introduction}

Understanding the structure of hadrons in terms of its fundamental constituents, quarks and gluons, driven by the strong interaction is one of the outstanding issues in hadron physics. The electromagnetic probes are an important tool to investigate the dynamics of Quantum Chromodynamics (QCD) in the perturbative and non-perturbative regime and they continue to make important contributions to our understanding of hadron structure today (for a review, see [1]). The small electromagnetic coupling of Quantum ElectroDynamics (QED) allows for a clean separation of the reaction mechanisms and the structure aspects which has led to factorization allowing for the extraction of non-perturbative structure objects of hadrons. The proton has been the subject of intensive studies since it is the lightest stable hadron. However, a complete understanding of its structure is yet to be found. For example, the proton radius, measured in muonic hydrogen atoms, has given a value that is 7 standard deviations lower than the values obtained from electronic hydrogen spectroscopy and elastic electron proton scattering [2].

Nucleon structure investigations have been performed since lepton beams became available. Elastic electron proton scattering has been intensively studied to determine the electromagnetic form factors of the proton in the region of negative 4-momentum transfer squared $\left(q^{2}<0\right.$, space-like region) [3]. At high energy, one can define a hard scale as "high $Q^{2}=-q^{2}$ " so that the factorization property of the scattering amplitude can be assumed. Factorization

\footnotetext{
*e-mail: adbeyssi@uni-mainz.de
} 
theorems, derived for several classes of hard electromagnetic processes, allow one to factorize the amplitudes into a perturbative hard part and a universal non-perturbative hadronic matrix element containing structure functions such as the parton distribution functions (PDFs), generalized parton distributions (GPDs), transverse momentum dependent parton distribution functions (TMD PDFs), and transition distribution amplitudes (TDAs). Extensive measurements of hard (semi-)inclusive and exclusive processes have been performed using high energy lepton beams [4] providing large, but not complete, amount of data on these nonperturbative structure functions.

Lepton scattering experiments allow for the access of nucleon structure matrix elements in the region of negative momentum transfer squared $q^{2}$ of the intermediate virtual photon. Experiments accessing the region of positive, time-like momentum transfer squared $q^{2}$ of the virtual photon, complement the studies of the nucleon structure. In this context, the future antiproton beams of momentum up to $15 \mathrm{GeV} / c$ at the Facility for Antiproton and Ion Research (FAIR) will push back the frontiers of the accessible kinematics and achievable precision. The PANDA experiment [5] at FAIR offers a unique possibility to study the proton structure in time-like region with different electromagnetic final channels. The physics program of PANDA includes for example measurements of the time-like electromagnetic from factors, the generalized distribution amplitudes (GDAs) which are the counterpart of the GPDs in the time-like region, the TDAs, and the TMD PDFs. The possibility to extract the same non-perturbative hadronic matrix element, like for the case of TDAs and TMD PDFs, from various high energy processes and in different kinematical regimes will give experimental support to these non-perturbative approaches.

\section{The PANDA experiment at FAIR}

The PANDA experiment is one of the keys projects planned at FAIR which is currently under construction at Darmstadt, Germany. The PANDA experiment will measure the annihilation reactions induced by a high-intensity antiproton beam with momenta from 1.5 to $15 \mathrm{GeV} / c$. The PANDA detector will be installed at the High Energy Storage Ring (HESR) of FAIR where first antiproton beams are expected in 2025. It is designed to provide large acceptance, high resolution and tracking capability and good neutral and charged particle identification in a high rate environment. The physics program includes wide range of physics topics like hadron spectroscopy including charmonium and open charm states, light mesons and baryons and QCD exotics, hypernuclear physics, study of hadron properties in nuclear medium, nucleon structure,... In the following, the physics goals related to the measurements of the proton electromagnetic form factors and the TDAs, are described.

\section{The proton electromagnetic form factors}

Electromagnetic form factors are fundamental quantities, which describe the electric and magnetic distributions of hadrons. Proton (spin 1/2 particle) is characterized by the electric $G_{E}\left(q^{2}\right)$ and the magnetic $G_{M}\left(q^{2}\right)$ form factor. They are experimentally accessible through measurements of differential cross sections for elastic electron proton scattering in the spacelike region and annihilation reactions as $e^{+} e^{-} \leftrightarrow \bar{p} p$ in the time-like region. It is assumed that the interaction occurs through the exchange of one photon, which carries the momentum transfer squared $q^{2}$. Proton form factors have been measured in space-like region since more than 60 years with increasing accuracy $[1,3]$. The recent data from the JLab-GEp collaboration (see [6] and references therein), based on the polarization transfer method [7] in elastic electron proton scattering, showed surprisingly that the ratio $\mu_{p} G_{E} / G_{M}\left(\mu_{p}\right.$ is the 
proton magnetic moment) decreases almost linearly with $Q^{2}$. This result is in contrast to the previous measurements of unpolarized elastic ep scattering. In the time-like region, the precision of the proton form factor measurements at the $e^{+} e^{-}$colliders and from $\bar{p} p$ annihilation experiments has been limited by the statistics. While the total cross section of the $e^{+} e^{-} \rightarrow \bar{p} p$ has been measured up to high values of $q^{2}\left(\sim 42(\mathrm{GeV} / c)^{2}\right)$, few data exist on the time-like proton form factor ratio in the region below $q^{2}=9(\mathrm{GeV} / c)^{2}$. The later has been measured by PS170 at LEAR [8], BABAR [9] and more recently by BESIII [10] and CMD-3 [11]. The results of PS170 and BABAR disagree with each other with a significance up to $3 \sigma$, while the BESIII and CMD-3 measurements have large total uncertainties.

The PANDA experiment will allow the measurement of the proton form factors in the time-like region with the processes $\bar{p} p \rightarrow \ell^{+} \ell^{-},(\ell=e, \mu)$ over a large kinematical range [12-14]. It will be the first time that muons in the final state will be used to measure the time-like form factors of the proton [15]. Moreover, the unphysical region below the kinematical threshold of the proton antiproton production of $\left(2 M_{p}\right)^{2}$ can be accessed through the measurement of the $\bar{p} p \rightarrow \ell^{+} \ell^{-} \pi^{0}$ process [16-19]. This region has never been experimentally accessed. The PANDA experiment can provide unique measurements of the proton form factors in this region [20]. In addition, since the time-like proton form factors are complex functions of the momentum transfer squared $q^{2}$, an experiment with a polarized antiproton beam and/or polarized proton target would allow access to the phase difference between $G_{E}$ and $G_{M}[21,22]$. The feasibility of implementing a transversely polarized proton target in PANDA detector is currently under investigation [23].

\subsection{Proton form factor measurements with the $\bar{p} p \rightarrow e^{+} e^{-}$process at PANDA}

Feasibility studies of $\bar{p} p \rightarrow e^{+} e^{-}$for the measurements of time-like electromagnetic proton form factors at PANDA have been performed [13]. Two independent simulations have been carried out to verify the reproducibility of the results and to check systematic effects. The signal selection was performed based on the raw output of sub-detectors as well as particle identification algorithms. A suppression factor for the main background process $\bar{p} p \rightarrow \pi^{+} \pi^{-}$ at the order of $10^{8}$ has been achieved keeping a large and sufficient signal efficiency. The systematic uncertainties on the extracted proton form factors have been also evaluated. With an integrated luminosity of $2 \mathrm{fb}^{-1}$, the proton form factor ratio can be determined with expected statistical (total) uncertainties of about $1 \%(3 \%)$ at $q^{2}=5.4(\mathrm{GeV} / c)^{2}$ up to $50 \%$ at $13.9(\mathrm{GeV} / c)^{2}$ (Fig. 1). A separate measurement of $\left|G_{E}\right|$ and $\left|G_{M}\right|$ at PANDA is also possible.

\subsection{Proton form factor measurements with the $\bar{p} p \rightarrow \mu^{+} \mu^{-}$process at PANDA}

The channel $\bar{p} p \rightarrow \mu^{+} \mu^{-}$has been also studied at four beam momenta between 1.5 and 3.3 $\mathrm{GeV} / c$ [14], complementing the feasibility studies of the $\bar{p} p \rightarrow e^{+} e^{-}$channel. A method based on multivariate data classification (Boosted Decision Trees) was used to optimize the separation of the signal from the main background channel $\bar{p} p \rightarrow \pi^{+} \pi^{-}$. Signal to background ratios between 1:5 and 1:13 (background rejection factor of $\sim 10^{-5}$ ) are achieved. A subtraction of the residual background events was applied in the analysis. After the background subtraction, the proton form factors are extracted from fitting the efficiency corrected angular distributions of the $\bar{p} p \rightarrow \mu^{+} \mu^{-}$channel. The achievable precisions of $\left|G_{E}\right|,\left|G_{M}\right|$ and $R=\left|G_{E}\right| /\left|G_{M}\right|$ are studied assuming an integrated luminosity of $2 \mathrm{fb}^{-1}$ per beam momentum setting and including both statistical and systematical uncertainties. The results of the simulations show that the proton form factors can be measured with the $\bar{p} p \rightarrow \mu^{+} \mu^{-}$channel at PANDA with a good precision. A total relative uncertainty on the measurement of the proton form factor ratio between $5 \%$ at $1.5 \mathrm{GeV} / c$ and $37 \%$ at $3.3 \mathrm{GeV} / c$ is expected. 


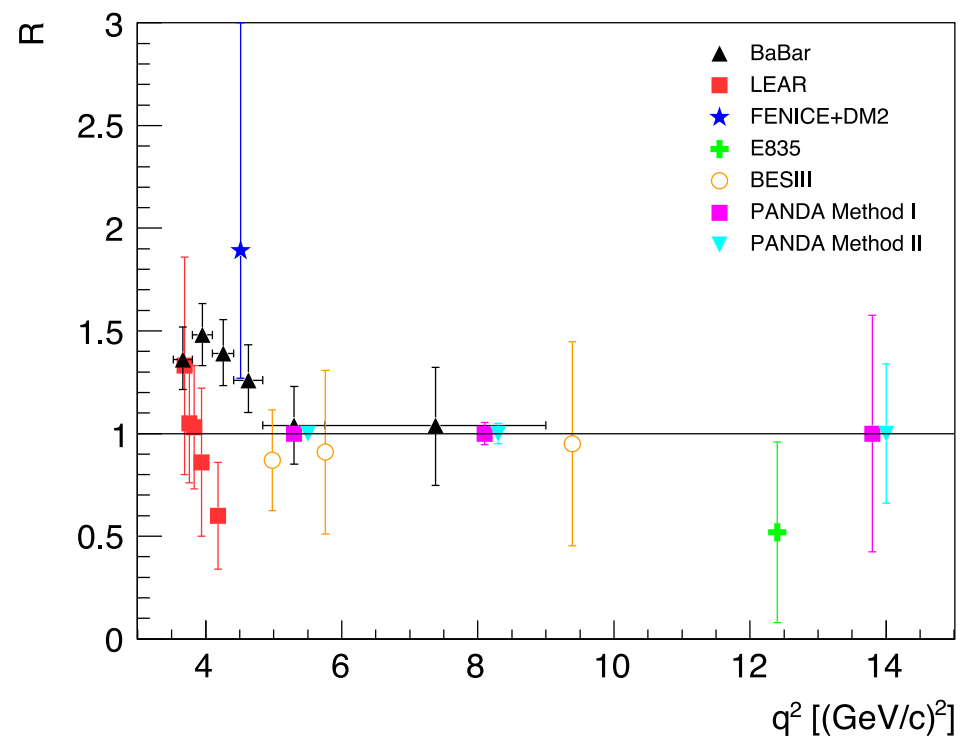

Figure 1. Expected statistical precision of the determination of the proton form factor ratio $R=$ $\left|G_{E}\right| /\left|G_{M}\right|$ at PANDA as a function of $q^{2}$, compared with the existing data (see [13] and references therein)

\section{The baryon-to-meson Transition Distribution Amplitudes}

Baryon-to-meson TDAs [24, 25] were introduced as a further generalization of the GPD concept for a new class of hard electromagnetic processes in which a description, similar to the collinear factorization theorem for hard meson electroproduction [26] can be applied. Baryon-to-meson TDAs describe partonic correlations inside nucleons. Fourier transforming TDAs to the impact parameter space allows one to perform femto-photography of hadrons. In particular, nucleon-to-meson $(\pi N)$ TDAs may be used as a tool for spatial imaging of the pion cloud inside the nucleon. Nucleon-to-pion TDAs arise in the description of several hard exclusive reactions such as backward electroproduction of pions off nucleons [27, 28], which can be in principle studied at JLab and COMPASS in the space-like regime. The PANDA experiment offers possibilities to access the same non-perturbative functions in the timelike regime [29-31], complementing the results obtained from the measurements of lepton beam induced reactions. Recently, detailed studies of the access to $\pi N$ TDAs in the reactions $\bar{p} p \rightarrow \gamma^{*} \pi^{0} \rightarrow e^{+} e^{-} \pi^{0}$ and $\bar{p} p \rightarrow J / \Psi \pi^{0} \rightarrow e^{+} e^{-} \pi^{0}$ in specific kinematic regimes (large $q^{2}$ and small $|t|=\left|\left(p_{\pi}-p_{\bar{p}}\right)^{2}\right|$ or small $\left.|u|=\left|\left(p_{\pi}-p_{p}\right)^{2}\right|\right)$ have been presented by the PANDA collaboration in [32, 33]. The channel $\bar{p} p \rightarrow \gamma^{*} \pi^{0} \rightarrow e^{+} e^{-} \pi^{0}$ has never been measured, however some data exist for $\bar{p} p \rightarrow J / \Psi \pi^{0} \rightarrow e^{+} e^{-} \pi^{0}[34,35]$ which have been used to constrain the predictions for PANDA.

The feasibility of measuring the $\bar{p} p \rightarrow \gamma^{*} \pi^{0} \rightarrow e^{+} e^{-} \pi^{0}$ process with the PANDA detector has been performed at center of mass energy squared $s=5 \mathrm{GeV}^{2}$ and $s=10 \mathrm{GeV}^{2}$ in the kinematic regimes where the description of this process in terms of TDAs is assumed. Assuming an integrated luminosity of $2 \mathrm{fb}^{-1}$, the results show that the differential cross section in bins of $q^{2}$ can be measured at PANDA with an averaged statistical uncertainty of $12 \%$ at $s=5 \mathrm{GeV}^{2}$, and $24 \%$ at $s=10 \mathrm{GeV}^{2}$ (Fig. 2). 
The $\bar{p} p \rightarrow J / \Psi \pi^{0} \rightarrow e^{+} e^{-} \pi^{0}$ process has been investigated at three values of the center of mass energy squared $s=12.3 \mathrm{GeV}^{2}, s=16.9 \mathrm{GeV}^{2}$ and $s=24.3 \mathrm{GeV}^{2}$. The resonant case presents the advantage of a larger cross section than the $\bar{p} p \rightarrow \gamma^{*} \pi^{0} \rightarrow e^{+} e^{-} \pi^{0}$. The statistical uncertainties to measure the differential cross section as a function of the four momentum transfer in the two relevant kinematics are expected to be about $8-10 \%$. The measurement of both resonant and non-resonant channels at PANDA allows one to test the universality of $\pi N$ TDAs in different kinematic ranges. In addition, the investigation of the baryon-to-photon TDAs will be possible at PANDA with the annihilation process $\bar{p} p \rightarrow \gamma^{*} \gamma \rightarrow e^{+} e^{-} \gamma$ [25]. Feasibility of measurement of the $\bar{p} p \rightarrow \gamma^{*} \gamma \rightarrow e^{+} e^{-} \gamma$ process at PANDA is planned.
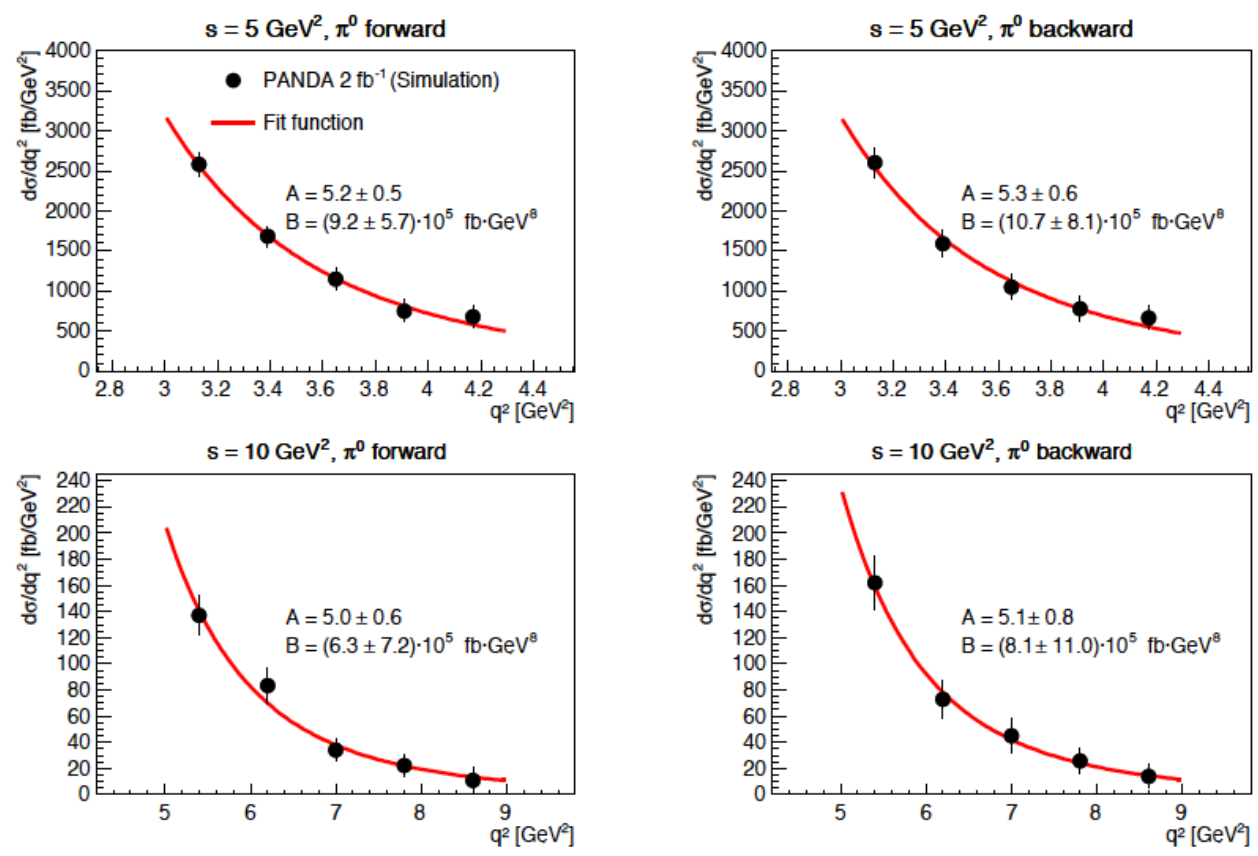

Figure 2. The $\bar{p} p \rightarrow \gamma^{*} \pi^{0} \rightarrow e^{+} e^{-} \pi^{0}$ differential cross sections as a function of $q^{2}$ obtained from the simulations for $s=5 \mathrm{GeV}^{2}$ and $s=10 \mathrm{GeV}^{2}$, in both the $t-\left(\pi^{0}\right.$ forward $)$ and the $u-\left(\pi^{0}\right.$ backward $)$ channel kinematic regimes [32]. The resulting cross section are fitted with the theoretical leading twist predictions (see [32] and references therein). The parameters A and B are fit parameters

\section{Conclusion}

The future PANDA experiment has a large and very competitive physics program to investigate the structure of the nucleon using electromagnetic processes with antiproton beams. Feasibility studies of measuring these processes with the PANDA detector have been performed, or are ongoing, using Monte Carlo simulations. In this contribution the studies related to the measurements of the time-like proton form factors and the TDAs at PANDA were presented. The results show the possibility to suppress the different background contributions to very low and sufficient levels and to extract the signal channels with good efficiencies. The PANDA experiment will allow the measurements of the proton form factors in the time-like region with high accuracy over a large and unexplored kinematical range. The resulting precisions 
on the measurements of the $\bar{p} p \rightarrow \gamma^{*}($ or $J / \Psi) \pi^{0} \rightarrow e^{+} e^{-} \pi^{0}$ differential cross sections will allow us in one side to test and constraint the TDA models, and on the other side to measure the proton form factors in the time-like unphysical region. In addition, the precise measurements of the structure functions such as the GDAs and TMD PDFs at PANDA [5] together with planned experiments at other facilities, will provide deep insight about the internal structure of the nucleon.

\section{References}

[1] S. Pacetti, R. Baldini Ferroli, and E. Tomasi-Gustafsson, Phys. Rept. 550-551, 1 (2015)

[2] R. Pohl et al., Nature 466, 213-216 (2010)

[3] A. J. R. Puckett, Phys. Rev. C. 96, 055203 (2017)

[4] M. Guidal, H. Moutarde, and M. Vanderhaeghen, Rept. Prog. Phys. 76, 066202 (2013)

[5] W. Erni et al., arXiv:0903.3905 [hep-ex]

[6] A. Puckett et al., Phys. Rev. Lett. 104, 242301 (2010)

[7] A. I. Akhiezer and M.P. Rekalo, Sov. Phys. Dokl. 13, 572 (1968)

[8] G. Bardin et al., Nucl. Phys. B 411, 3-32 (1994)

[9] J. P. Lees et al., Phys. Rev. D 88, 072009 (2013)

[10] M. Ablikim et al., Phys. Rev. D 91, 112004 (2015)

[11] R. R. Akhmetshin et al., Phys. Lett. B 759, 634-640 (2016)

[12] M. Sudol et al., Eur. Phys. J. A 44, 373-384 (2010)

[13] B. Singh et al., Eur. Phys. J. A 52, 325 (2016)

[14] I. Zimmermann, Feasibility studies for the measurement of the time-like electromagnetic proton form factors at the PANDA experiment. PhD thesis (Johannes Gutenberg University, 2017)

[15] A. Dbeyssi, E. Tomasi-Gustafsson, G. I. Gakh, and M. Konchatnyi, Nucl. Phys. A 894, 20-40 (2012)

[16] M. P. Rekalo, Sov. J. Nucl. Phys. 1, 760 (1965)

[17] A. Z. Dubnickova, S. Dubnicka, and M. P. Rekalo, Z. Phys. C 70, 473 (1996)

[18] C. Adamuscin, E. A. Kuraev, E. Tomasi-Gustafsson, and F. E. Maas, Phys. Rev. C 75, 045205 (2007)

[19] J. Guttmann and M. Vanderhaeghen, Phys. Lett. B 719, 136-142 (2013)

[20] J. Boucher, Feasibility studies of the $\bar{p} p \rightarrow e^{+} e^{-} \pi^{0}$ electromagnetic channel at PANDA. $\mathrm{PhD}$ thesis (University of Paris-Sud XI and of the Johannes Gutenberg University, 2011)

[21] A. Z. Dubnickova, S. Dubnicka, and M. P. Rekalo, Nuovo Cim. A 109, 241-256 (1996)

[22] E. Tomasi-Gustafsson, F. Lacroix, C. Duterte, and G. I. Gakh, Eur. Phys. J. A 24, 419 (2005)

[23] B. Froehlich, Investigation on intense magnetic flux shielding with a high temperature superconducting tube for a transverse polarized target at the PANDA experiment. $\mathrm{PhD}$ thesis (Johannes Gutenberg University, 2017)

[24] L. L. Frankfurt, P. V. Pobylitsa, Maxim V. Polyakov, and M. Strikman, Phys. Rev. D 60, 014010 (1999)

[25] B. Pire and L. Szymanowski, Phys. Rev. D 71, 111501 (2005)

[26] J. C. Collins, L. Frankfurt, and M. Strikman, Phys. Rev. D 56, 2982-3006 (1997)

[27] J. P. Lansberg, B. Pire, and L. Szymanowski, Phys. Rev. D 75, 074004 (2007)

[28] J. P. Lansberg, B. Pire, K. Semenov-Tian-Shansky, and L. Szymanowski, Phys. Rev. D 85, 054021 (2012) 
[29] J. P. Lansberg, B. Pire, and L. Szymanowski, Phys. Rev. D 76, 111502 (2007)

[30] J. P. Lansberg, B. Pire, K. Semenov-Tian-Shansky, and L. Szymanowski, Phys. Rev. D 86, 114033 (2012)

[31] B. Pire, K. Semenov-Tian-Shansky, and L. Szymanowski, Phys. Lett. B 724, 99-107 (2013)

[32] B. P. Singh et al., Eur. Phys. J. A 51, 107 (2015)

[33] B. Singh et al., Phys. Rev. D 95, 032003 (2017)

[34] T. A. Armstrong et al., Phys. Rev. Lett. 69, 2337-2340 (1992)

[35] M. Andreotti et al., Phys. Rev. D 72, 032001 (2005) 